\section{ACCURATE SCANNING WITH LESS RADIATION}

Until recently, implant specialists have used CT (computed tomography) or CAT (computed axial tomography) scanning in situations where 3-D imaging helps formulate a diagnosis. Traditional CT uses a narrow fan beam that rotates around the patient acquiring thin axial slices with each revolution.

In order to image a section of anatomy, many rotations must be completed which leads to a higher dose of X-ray radiation and also might leave a gap or break in information between each rotation. The imaging software must fill in or extrapolate the missing information.

The NewTom differs from a traditional hospital CT scan in the way it captures an image. The X-ray tube revolves around the patient's head emitting a cone-shaped beam to acquire the entire image set in a single rotation, capturing a series of 360 flat panel images, one for each of the 360 degrees it rotates.

A dental CT scan takes ten minutes and exposes the patient to two minutes of radiation; a NewTom scan takes just 18 seconds and because it uses SafeBeam technology and pulsed $\mathrm{X}$-ray emission, it exposes the patient to 3.6 seconds of low-dose radiation.

NewTom Cone Beam 3D Imaging Systems give more information than conventional CT scans meaning greater accuracy in detection and diagnosis. Reader response number 60

\title{
REINVENTING IMAGE PLATE WORKFLOW
}

The new CS 7600 from Carestream Dental is a compact, cost-effective intraoral imaging plate system that delivers high image quality, quickly, and includes an exclusive automated and secured workflow for superior ease of use.

The first plate system to include Scan ct Go technology, CS 7600 captures superior intraoral images, such as those achieved using film, but with faster and more consistent results. Images can even be prioritised by speed or resolution, based on diagnostic needs.

A simple and affordable way to make the move to digital imaging, the system's advanced plate imaging technology integrates smoothly into any environment. A compact design ensures the unit will fit practically anywhere, making it a great investment for small and large practices alike.

Fully automated technology not only eliminates waiting time but also simplifies switching between patients - and all while eliminating the risk of image mix-ups and confusion.

The CS 7600 has reinvented imaging plate workflow without the need for dental professionals to change their working habits. CS 7600 offers all the benefits of digital imaging, creating high resolution images in seconds and is a cost-effective option for all. Reader response number 61

\section{KEEPING THE MOUTH MOIST}

The OralSeven range of oral care products can help relieve the symptoms of the auto-immune condition Sjögren's syndrome.

Sjögren's syndrome affects nearly half a million people in the UK and is very unpleasant and uncomfortable. The secretory glands stop working which leads to a loss of saliva and dry eyes, skin and gastrointestinal tract; it has a devastating effect on the lives of those with the condition - mainly women between the ages of 30-60.

There is no cure for the condition, but there are ways to help relieve the symptoms such as the lack of saliva. The OralSeven range of oral care products contain natural enzymes to assist the functions of healthy saliva, thus keeping the mouth moist and balanced. Maintaining oral hydration can be a problem as many of the conventional dental products contain either alcohol in the case of mouthwashes or sodium lauryl sulphate (SLS) in toothpaste, both of which have a drying effect and will only make the problem worse.

The range includes: OralSeven Toothpaste - with no harsh drying or SLS; OralSeven Mouthwash - with no alcohol; and OralSeven Moisturising Mouth Gel - which soothes, lubricates and protects the mouth and gums for up to seven hours.

\section{Reader response number 62}

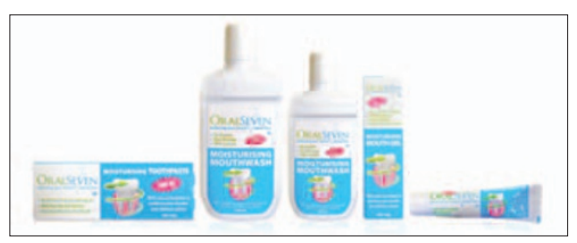

\section{FACILITATING A FAST RECOVERY}

Having implants fitted is both emotionally and physically draining for patients and they are naturally upset at the prospect that, having undergone such invasive treatment, they are obliged to further aggravate the sensitive treatment site by cleaning it fastidiously to stave off infection.

Practitioners can ease the burden on their patients by recommending the
Waterpik Nano Water Flosser, which cleans implants effectively without rubbing or scraping the delicate post treatment tissue.

Waterpik Water Flossers have been clinically proven to be twice as effective as traditional floss in the improvement and maintenance of oral health and are ideal for patients for whom the use of traditional floss would prove difficult.

The Waterpik Nano Water Flosser is smaller and quieter than previous models and comes with three pressure settings to suit various levels of sensitivity, making it the perfect solution before, during and after treatment - and it is the first Water Flosser to be powered from a shaver socket.

For implant patients, interdental cleaning with the Waterpik Nano Water Flosser is quick and easy and facilitates a fast recovery. Reader response number 63 